\title{
How Important Is Context in Teaching Interviewing?
}

$\mathbf{R}$ esearchers necessarily make assumptions about the phenomena they study. These assumptions frame the questions they ask and the methods used to answer them. In this editorial, I contrast two sets of assumptions, or models, of social action and show the implications each model has for teaching interviewing in medicine.

In the first model of social action, researchers assume that individuals have abstract and generalized beliefs that are relatively stable and stand apart from concrete situations. Individuals select communication practices and courses of action that are consistent with these beliefs. The more strongly an individual holds a set of beliefs, the more likely it will influence his or her choices.

This model is exemplified by Levinson and Roter's study in this issue of the Journal. ${ }^{1}$ Levinson and Roter assessed the relationship between physicians' beliefs about psychosocial aspects of patient care and their communication with patients. They treated the physician as the unit of analysis. They assigned a score to each physician to reflect that physician's attitudes about the importance of pyschosocial issues. They found that physicians who believed in the importance of pyschosocial aspects in medical interviews used fewer closedended questions and more statements of emotions than did their colleagues who attached less importance to psychosocial matters.

In the second model of social action, one which has gained popularity in various disciplines, including medicine, psychology, sociology, anthropology, and communication sciences, individuals decide how to communicate based on the characteristics of the current interaction. ${ }^{2}$ This model moves in closer to the perspective of the participants and travels with them in time as they interact. In responding to what a patient just said or did, for example, a physician attempts to satisfy institutional demands, deal with numerous concerns, and honor multiple beliefs and commitments.

Using this model, a researcher might ask: What are the issues involved and the concerns experienced by the physician at different points in an interview (for example, when opening an interview, when responding to an emotional patient, or when delivering bad news)? What are the communicative choices available to the physician at different points in the interview? How do communication choices affect the interaction? Researchers might design studies to identify the concerns of the participants, their alternatives for action, and the interactional consequences of the alternatives within conversational contexts.
There are various research techniques that are compatible with this model of social interaction. Conversation-analytic methods allow researchers to examine the strategies physicians select in local interactional environments and to track the interactional consequences of their selections. A variety of ethnographic techniques, including stimulated recall and focus group interviews, provide researchers with the opportunity to explore both physicians' and patients concerns as they, themselves, identify them. Combining research techniques permits researchers to associate the participants' expressed concerns with the communication occurring at those points.

What are the implications each model has for teaching interviewing in medicine? Operating with the first model, researchers might find that specific sets of beliefs of physicians are associated with their use of specific communication strategies. They might conclude that teachers should inculcate students with specific sets of beliefs so as to influence their conduct. Levinson and Roter, for example, recommend that medical educators reflect on how they might influence the beliefs of their students concerning the importance of psychosocial issues in patient care. They suggest that the way to change physicians' interviewing behavior is to change their beliefs.

The pedagogical implications of the second model for teaching interviewing in medicine are twofold. First, instruction might be aimed at increasing students' understanding of the role of context in the communication process and students' ability to produce and make use of different interactional contexts. Second, teachers might directly teach those communication skills that are needed to handle the problems that the contextural analyses identify.

By now, my preference for the second model of social action should be transparent. Meaning lies in words in context, not in words themselves. To understand and teach communication skills, we need a model of social interaction that incorporates context as a key component.-Anita Pomerantz, PhD, Associate Professor, Department of Communication Sciences, Temple University, Philadelphia, PA 19122

\section{REFERENCES}

1. Levinson W, Roter D. Physicians' psychosocial beliefs correlate with their patient communication skills. J Gen Intern Med. 1995; 10:3759 .

2. Sacks H. Lectures on Conversation. Vols 1 and 2. Oxford, UK: Black well. 1992 . 